\title{
Anisotropy of incommensurate spin and charge fluctuations in detwinned $\mathrm{YBa}_{2} \mathrm{Cu}_{3} \mathrm{O}_{6+\delta}$
}

\author{
T. Zhou ${ }^{1}$ and Jian-Xin $\mathrm{Li}^{1,2}$ \\ ${ }^{1}$ National Laboratory of Solid State of Microstructure and Department of Physics, \\ Nanjing University, Nanjing 210093, China \\ ${ }^{2}$ The Interdisciplinary Center of Theoretical Studies, \\ Chinese Academy of Science, Beijing 100080, China.
}

(Dated: May 7, 2005)

\begin{abstract}
Motivated by a recent neutron scattering experiment on detwinned $\mathrm{YBa}_{2} \mathrm{Cu}_{3} \mathrm{O}_{6+\delta}$ superconductor, we examine the frequency and doping dependence of the anisotropy in the spin and charge fluctuation arising from the coupling between the plane and the chain. Starting from the twodimensional $t-t^{\prime}-J$ model and using the random-phase approximation (RPA), we find a pronounced anisotropy of the incommensurate (IC) peaks in the spin channel, namely the peak intensity at the $(\pi \pm \delta, \pi)$ direction is stronger than that at the $(\pi, \pi \pm \delta)$ direction in a wide frequency range from $\omega=0.2 J$ to the resonance frequency $\omega_{r}=0.35 J$. Above the resonance frequency, the IC peaks reemerge. Their intensities shift to the diagonal direction and no anisotropy exists along the two diagonal directions. We find that this anisotropy is robust with respect to the possible variation of the RPA correction factor and to the dopings. The charge fluctuation is also found to be incommensurate for all energies considered and peak at $(0, \delta)$ and $(\delta, 0)$. An anisotropy in its IC peak intensity along the $k_{x}$ and the $k_{y}$ direction exists, but in sharp contrast to the spin channel, the maximum intensity of the IC peak is along the $k_{y}$ direction. Moreover, the IC peak in the charge channel exhibits an upward dispersion, in contrast to the downward dispersion below the spin resonance frequency for the spin IC peak. We explain these features based on the effect of the plane-chain coupling on the topology of the Fermi surface.

PACS numbers: 74.72.Bk, 74.25.Ha, 74.20.Mn
\end{abstract}

\section{INTRODUCTION}

The spin and charge dynamics are important issues in the studies of the high- $T_{c}$ superconductivity. The inelastic neutron scattering (INS) experiments have been playing important roles on studying their spin dynamics. It can provide direct information of the momentum and frequency dependence of the dynamical spin susceptibility. On the other hand, the INS experiments can measure the charge fluctuations indirectly by observing a change in the mass density. In addition to the indirect measurement, the charge susceptibility can be detected directly by the inelastic x-ray or electron scattering.

Over the past decade, the INS experiments carried out on the twinned samples have observed the four-fold symmetric incommensurate (IC) peaks at the wave vector $\mathbf{q}=(\pi, \pi \pm \delta)$ and $\mathbf{q}=(\pi \pm \delta, \pi)$ in a range of low energies [1, 2, 3], in addition to a sharp commensurate resonance peak around the antiferromagnetic $(\mathrm{AF})$ wave vector $\mathbf{Q}=(\pi, \pi)$ at about $41 \mathrm{meV}$ [, 5, 6]. The experiment also reported that the IC peaks at $(0, \pm 2 \delta)$ are observed for the charge excitation through the measurement of atomic mass density change [7, 8]. Theoretically, there are two possible explanations of the spin incommensuration in the INS experiments. One is based on the stripedphase picture, which suggests that the presence of the dynamic stripes is the origin of the IC peaks 9,10$]$. The other is the scenario of the nested-Fermi-surface which can explain the gross feature of the incommensurate spin fluctuations and spin resonance 11, 12, 13, 14, 15, 16, 17.
Recently, Mook et al. have carried out the neutron scattering measurement on partly detwinned $\mathrm{YBa}_{2} \mathrm{Cu}_{3} \mathrm{O}_{6.6}[18]$ and found that the IC peak shows the one dimensional (1D) feature. They claimed that it gives a strong support on the scenario of the stripe-phase picture. However, we showed that [19] an anisotropic IC peak can in fact be obtained when the coupling between the plane and chain, and the asymmetry of the crystal lattice between the $a$ and $b$ axis are considered in the two-dimensional $t-t^{\prime}-J$ model, based on the Fermi surface topology. Very recently, an INS experiment on fully detwinned YBCO [20] shows that the geometry of the magnetic fluctuations is actually two-dimensional, but with a prominent anisotropy in the amplitude and the width of the IC peak which is maximum along the $(q, \pi)$ direction and the anisotropy is strongly energy-dependent. Our previous theoretical calculation [19] shows many features which are consistent with the experimental data [20]. However, the linewidth anisotropy found in the experiment is more stronger than our calculation at the fixed energy $\omega=0.3 J(J$ is the AF coupling constant in the $t-t^{\prime}-J$ model) and a detail theoretical investigation of the energy dependence of the linewidth anisotropy as found experimentally still lacks [21]. Another new development in recent INS experiments is the reemergence of the IC peak at higher energies above the spin resonance energy 22, 23]. This high-energy IC peak is found to shift from the $(\pi, q)$ (and its symmetric points) direction to the diagonal direction [22, 23]. This reemergence of the spin incommensuration has in fact been predicted theoreti- 
cally based on the scenario of the Fermi surface topology [13, 14, 15]. However, both the experimental and theoretical investigations are carried out in the twinned systems. So, an interesting issue is how the IC peak behaves in this high-energy region in the detwinned systems.

Though we have shown before that the plane-chain coupling, and the asymmetry of the crystal structure can lead to the in-plane anisotropy of the spin IC peak, their effect may differ in detail 19]. Namely, the former leads the maximum IC peak to be along the $k_{x}$ direction, but the latter be along the $k_{y}$ direction. Due to the much stronger anisotropy caused by the planechain coupling, the combined effect gives rise to a maximum IC peak along the $k_{x}$ direction, which is consistent with experiments [18, 20]. Our investigation is based on a self-consistent calculation of the quasiparticle dispersion and the superconducting (SC) gap in the framework of the slave-boson approach to the $t-t^{\prime}-J$ model. We note that a recent calculation based on the similar RPAtype theory with the phenomenological quasiparticle dispersion and SC gap obtained by fitting to experiments shows that the asymmetric crystal structure also leads to the $k_{x}$-direction IC peak [24], in contrast to what we obtained before. We find that this contrasting behavior comes from the different relative ratios between the $a-b$ anisotropy of the nearest-neighbor hopping constant and the SC gap asymmetry, both are caused by the orthorhombic crystal structure. The asymmetric SC gap leads to a $k_{y}$-direction IC peak, while the anisotropy in the hopping constant leads to a $k_{x}$-direction IC peak. Our self-consistent calculation gives a $\Delta_{x} / \Delta_{y} \approx 1.5$ (the gap function is $\Delta_{\mathbf{k}}=\Delta_{x} \cos k_{x}-\Delta_{y} \cos k_{y}$ ), which is bigger than the value used by Eremin et al. 24]. So, the overwhelming asymmetric SC gap in our calculation leads to the anisotropy be in the $k_{y}$ direction. Therefore, the explanation of the anisotropy in the IC peak based on the orthorhombic crystal structure may depend on the fine tuning of the dispersion and SC gap, in particular its effect is shown to be much weaker than that from the plane-chain coupling [19]. So, we will just focus on the effect of the plane-chain coupling in this paper.

In this paper, we will first extend our previous scheme [19] to address the above issues in view of the new experimental results [20, 22, 23]. We will also examine in detail the effects on the anisotropic IC peaks of dopings and the RPA correction factor $\alpha$ (see the text below) [1], in order to check the robustness of this anisotropy. Our aim is not only to account for the new findings observed by the very recent experiment [20], but also to predict some new features which help to determine if the scenario of the nested-Fermi-surface can describe all features of the spin incommensuration or some exotic scenarios such as the stripe phase or the electronic nematic phase 25] may be required. In addition, we will also calculate the charge susceptibility and investigate the frequency dependence of the anisotropy in the charge channel.

The article is organized as follows. In Sec. II, we intro- duce the model and define notations. The Hamiltonian and formalism are obtained. In Sec. III, we present numerical results of the spin and charge susceptibility, respectively. In Sec. IV, we interpret the results. Finally, we present the conclusion in Sec. V.

\section{HAMILTONIAN AND FORMALISM}

We start with a Hamiltonian which describes the system with a $\mathrm{CuO}_{2}$ plane and a $\mathrm{CuO}$ chain per unit cell [19].

$$
H=H_{t-t^{\prime}-J}+H_{c}+H_{I}
$$

where $H_{t-t^{\prime}-J}$ is the two-dimensional $t-t^{\prime}-J$ Hamiltonian describing the $\mathrm{CuO}_{2}$ plane,

$$
\begin{aligned}
H_{t-t^{\prime}-J}= & -t \sum_{i \alpha \sigma} c_{i \sigma}^{\dagger} c_{i+\alpha \sigma}-h . c .-t^{\prime} \sum_{i \sigma} c_{i \sigma}^{\dagger} c_{i+\hat{x}+\hat{y} \sigma}-h . c \\
& +J \sum_{i \alpha}\left(\mathbf{S}_{i} \cdot \mathbf{S}_{i+\alpha}-\frac{1}{4} n_{i} n_{i+\alpha}\right)
\end{aligned}
$$

$H_{c}$ describes the $\mathrm{CuO}$ chain,

$$
H_{c}=-\sum_{i \alpha \sigma} t_{c} d_{i \sigma}^{\dagger} d_{i+\alpha \sigma}-\text { h.c. }
$$

and the coupling between the plane and chain is [26],

$$
\begin{aligned}
H_{I}= & -t_{\perp} \sum_{i j \sigma}\left(c_{i \sigma}^{\dagger} d_{j \sigma}+h . c\right)-\lambda / 4 \sum_{i \alpha}\left(\widehat{\Delta}_{1, i, \alpha}^{\dagger} \widehat{\Delta}_{2, i+\bar{c} / 2, \alpha}\right. \\
& \left.+\widehat{\Delta}_{1, i, \alpha}^{\dagger} \widehat{\Delta}_{2, i-\bar{c} / 2, \alpha}+\text { h.c }\right),
\end{aligned}
$$

where $c_{i \sigma}^{\dagger}$ and $d_{i \sigma}^{\dagger}$ are the creation operators of electrons in the plane and chain, respectively, $\alpha=\hat{x}, \hat{y}$ stands for unit lattice vector along the $a$ and $b$ axis, and $\mathbf{S}_{i}=\frac{1}{2} c_{i \alpha}^{\dagger} \sigma_{\alpha \beta} c_{i \beta} . \widehat{\Delta}_{n r \alpha}$ are the singlet pair operators, which are expressed by $\widehat{\Delta}_{1 r \alpha}=c_{r \uparrow} c_{r+\alpha \downarrow}-c_{r \downarrow} c_{r+\alpha \uparrow}$ and $\widehat{\Delta}_{2 r \alpha}=d_{r \uparrow} d_{r+\alpha \downarrow}-d_{r \downarrow} d_{r+\alpha \uparrow}$. Following Ref. [19], we use the slave-boson mean-field theory to the plane Hamiltonian $H_{t-t^{\prime}-J}$, in which the physical electron operators $c_{i \sigma}$ are expressed by slave bosons $b_{i}$ carrying the charge and fermions $f_{i \sigma}$ representing the spin, $c_{i \sigma}=b_{i}^{\dagger} f_{i \sigma}$. The mean-field Hamiltonian can be written in a matrix form [19, 27], and the mean-field order parameters $\Delta_{1 i j}=<f_{i \uparrow} f_{j \downarrow}-f_{i \downarrow} f_{j \uparrow}>= \pm \Delta_{1}, \Delta_{2 i j}=<$ $d_{i \uparrow} d_{j \downarrow}-d_{i \downarrow} d_{j \uparrow}>= \pm \Delta_{2}$ ( \pm depend on if the bond $<i j>$ is in the $\hat{x}$ or the $\hat{y}$ direction), $\chi_{i j}=\Sigma_{\sigma}<f_{i \sigma}^{\dagger} f_{j \sigma}>=\chi_{0}$, and the chemical potentials $\mu_{1}$ and $\mu_{2}$ for different planar doping densities $x$ can be obtained from the selfconsistent equations 19]. The doping densities for the chain are fixed to $x_{c h}=0.5$. The other parameters are choosed as $t=2 J, t^{\prime}=-0.45 t, \lambda=3 J$, the renormalized plane-chain hoping constant $\widetilde{t}_{\perp}=t_{\perp} \sqrt{x}=-0.1 \mathrm{~J}$, and the temperature $T=0.005 J$. The chain is along $k_{y}$ direction. 
The bare spin susceptibility $\chi_{0}^{s}(\mathbf{q}, \omega)$ coming from the particle-hole excitations of the planar fermions can be calculated from the two-particle Green's function $\chi_{0}^{s}(\mathbf{q}, i \omega)$ by the analytical continuation $i \omega \rightarrow \omega+i \delta$,

$$
\begin{aligned}
\chi_{0}^{s}(\mathbf{q}, \omega)= & \frac{1}{N} \sum_{\mathbf{k}} \sum_{i} \sum_{j}\left[U_{1 i}^{2}(\mathbf{k}) U_{1 j}^{2}(\mathbf{k}+\mathbf{q})\right. \\
& \left.+U_{1 i}(\mathbf{k}) U_{2 i}(\mathbf{k}) U_{1 j}(\mathbf{k}+\mathbf{q}) U_{2 j}(\mathbf{k}+\mathbf{q})\right] \\
& \times \frac{f\left(E_{j}(\mathbf{k}+\mathbf{q})\right)-f\left(E_{i}(\mathbf{k})\right)}{\omega-E_{j}(\mathbf{k}+\mathbf{q})+E_{i}(\mathbf{k})+i \delta} .
\end{aligned}
$$

with $U_{i j}$ are the elements of the matrix which diagonalizes the mean-field Hamiltonian [19].

The correction of the AF spin fluctuations to the spin susceptibility is included in the form of the renormalized random-phase approximation (RPA),

$$
\chi^{s}(\mathbf{q}, \omega)=\chi_{0}^{s}(\mathbf{q}, \omega) /\left[1+\alpha J_{\mathbf{q}} \chi_{0}^{s}(\mathbf{q}, \omega)\right] .
$$

Here $J_{\mathbf{q}}=J\left(\cos q_{x}+\cos q_{y}\right)$. The RPA correction factor $\alpha(\alpha<1)$ is introduced to overcome the overestimation of the AF correlation in the usual $(\alpha=1)$ RPA [11, 28]. Experimentally, the spin resonance peak is observed at $41 \mathrm{meV}$ at the optimal doping $x=0.14$ which corresponds to about $0.35 \mathrm{~J}$. Thus, whenever not be indicated, the $\mathrm{AF}$ correction factor $\alpha=0.5$ will be chosen to make the resonance frequency occur at $0.35 \mathrm{~J}$ at the optimal doping density $x=0.14$.

The bare charge susceptibility can also be calculated from the two-particle Green's function,

$$
\begin{aligned}
\chi_{0}^{c}(\mathbf{q}, \omega)= & \frac{1}{N} \sum_{\mathbf{k}} \sum_{i} \sum_{j}\left[U_{1 i}^{2}(\mathbf{k}) U_{1 j}^{2}(\mathbf{k}+\mathbf{q})\right. \\
& \left.-U_{1 i}(\mathbf{k}) U_{2 i}(\mathbf{k}) U_{1 j}(\mathbf{k}+\mathbf{q}) U_{2 j}(\mathbf{k}+\mathbf{q})\right] \\
& \times \frac{f\left(E_{j}(\mathbf{k}+\mathbf{q})\right)-f\left(E_{i}(\mathbf{k})\right)}{\omega-E_{j}(\mathbf{k}+\mathbf{q})+E_{i}(\mathbf{k})+i \delta} .
\end{aligned}
$$

In the form of RPA, the renormalized charge susceptibility can be written as

$$
\chi^{c}(\mathbf{q}, \omega)=\chi_{0}^{c}(\mathbf{q}, \omega) /\left[1-\frac{1}{4} J_{\mathbf{q}} \chi_{0}^{c}(\mathbf{q}, \omega)\right] .
$$

\section{THE NUMERICAL RESULTS}

\section{A. dynamical spin susceptibility}

Fig.1(a-c) show the intensity plot of the imaginary part of the spin susceptibility in the momentum space with doping $x=0.14$ at frequencies $0.3 \mathrm{~J}, 0.5 \mathrm{~J}$, and $0.75 \mathrm{~J}$, respectively. The IC peak intensity as a function of frequency at different momentum directions is shown in Fig.1(d). From Fig.1(a), one can see that the IC peaks show clear anisotropy between the $k_{x}$ and $k_{y}$ direction at $\omega=0.3 J$, namely, the incommensurability $\delta$ and the intensity of the IC peaks at $\left(\pi \pm \delta_{x}, \pi\right)$ is obviously larger
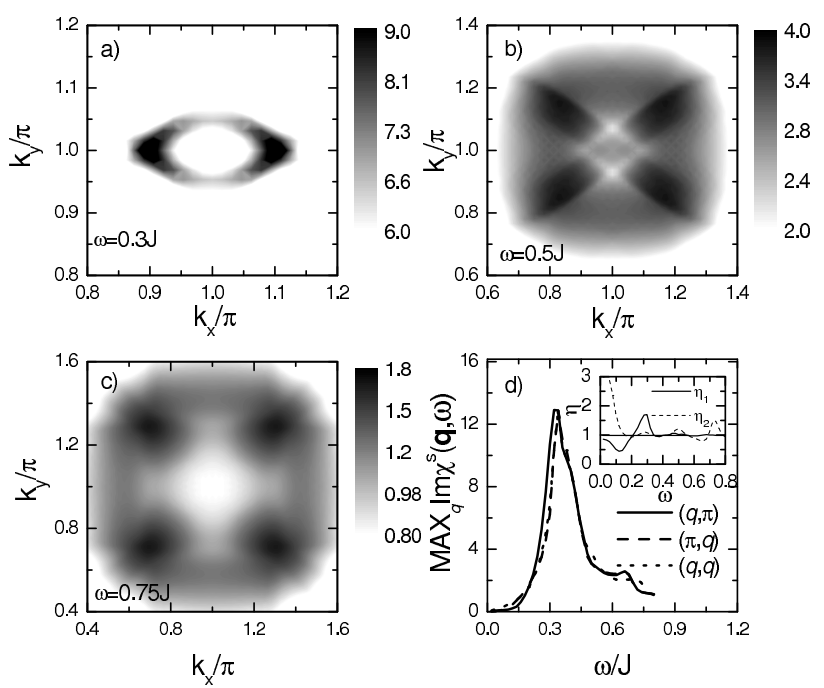

FIG. 1: Panels a)-c) are the intensity plot of the imaginary part of the spin susceptibility $\operatorname{Im} \chi^{s}$ as a function of the wave vector with doping $x=0.14$ for $\omega=0.3 \mathrm{~J}, 0.5 \mathrm{~J}$, and $0.75 \mathrm{~J}$, respectively. Panel d) shows the frequency dependence of the maximal intensity of $\operatorname{Im} \chi^{s}$. The inset of panel d) shows the ratio of the maximal $\operatorname{Im} \chi^{s}$ at different momentum directions (see text).

than that at $\left(\pi, \pi \pm \delta_{y}\right)$. When frequency is increased to be above the resonance frequency $0.35 \mathrm{~J}$, the IC peaks rotate to the diagonal direction, as is shown in Fig.1(b) and Fig.1(c). Interestingly, now no anisotropy exists along the two diagonal directions. The anisotropy below the resonance frequency is found to be frequency dependent, as shown in Fig.1(d). Detailed frequency dependence can be found in the inset of Fig.1(d), in which the ratio of the peak amplitude between the $k_{x}$ and $k_{y}$ direction $\left(\eta_{1}\right)$, and between the diagonal and the $k_{y}$ direction $\left(\eta_{2}\right)$, as a function of energy $\omega$ is presented $\left(\eta_{1}(\omega)=\max _{q} \operatorname{Im} \chi^{s}(q, \pi, \omega) / \max _{q} \operatorname{Im} \chi^{s}(\pi, q, \omega), \eta_{2}(\omega)=\right.$ $\left.\max _{q} \operatorname{Im} \chi^{s}(q, q, \omega) / \max _{q} \operatorname{Im} \chi^{s}(\pi, q, \omega)\right)$. As shown, the anisotropy is significant in a wide frequency range from slightly below the resonance frequency to about $0.2 \mathrm{~J}$. It increases firstly when the energy is reduced from the resonance and reaches the maximal value $\eta_{1}=1.7$ when frequency is at about $0.28 \mathrm{~J}$, then decreases with the further decrease of energy. The frequency dependence of the anisotropy is reasonably consistent with the experiment [20], in which the maximal ratio is about 2.1 at $\omega=28 \mathrm{meV}$. Below $0.2 \mathrm{~J}$, the IC peak rotates again to the diagonal direction as expected from the node-to-node excitations at low frequencies.

We now study the dependence of the anisotropy on the possible variations of the AF correction factor $\alpha$ with frequency $\omega=0.3 J$ and on doping density $x$ with frequency $\omega=0.25 \mathrm{~J}$ in Fig.2(a-b). The main panel shows the maximal intensity of spin excitations at different momentum directions and the inset is the ratio of the peak amplitude between the $q_{y}=\pi$ and the $q_{x}=\pi$ direction $\left(\eta_{1}\right)$. From Fig.2(a), one can see that the anisotropy is enhanced 

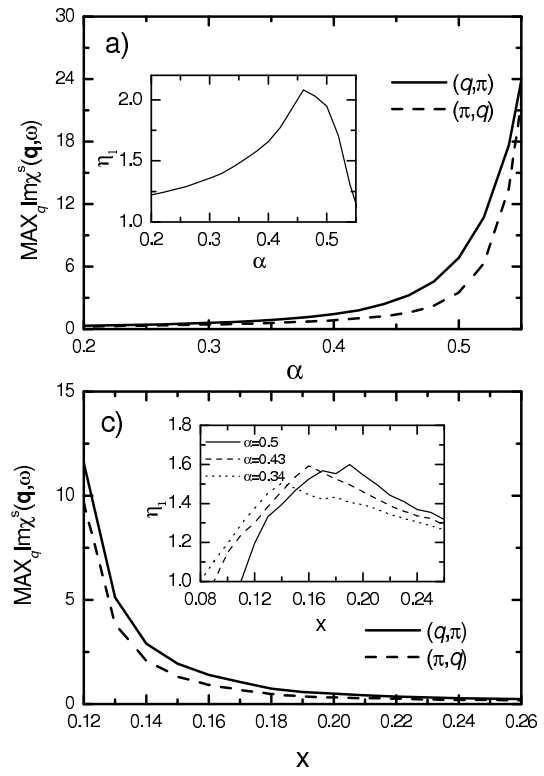

FIG. 2: Dependence of the maximal peak in $\operatorname{Im} \chi^{s}$ on the RPA correction factor $\alpha[\mathrm{a})]$ and doping $x[\mathrm{~b})]$, with $\omega=0.3 J$. The inset of figures shows the ratio of the maximal $\operatorname{Im} \chi^{s}$ between the $(q, \pi)$ and the $(\pi, q)$ direction.

gradually with an increasing of the AF correction factor $\alpha$. When $\alpha$ equals 0.47 , the anisotropy reaches its maximal value. Then, the further increase of $\alpha$ makes the anisotropy decrease and when $\alpha=0.57$, the anisotropy vanishes. As shown in Eq.(6), the AF correction factor $\alpha$ acts to enhance the AF correction, so the resonance peak is shifted to lower energies with the increase of $\alpha$. When $\alpha=0.57$, the spin resonance right appears at $\omega=0.3 J$, so the anisotropy vanishes because the spin resonance peaks at $(\pi, \pi)$. The critical doping density for the AF instability observed by experiments is $x_{c}=0.02 \sim 0.05$. It will give rise to $\alpha=0.34 \sim 0.43$ if one determine the AF correction factor $\alpha$ according to this experimental value $x_{c}$ [11, 15, 16. In this range of $\alpha$, the anisotropy is about $1.35 \sim 2.0$, as can be seen from the inset of Fig.2(a), so it is robust with respect to the possible variation of $\alpha$. The doping dependence of the anisotropy with frequency $\omega=0.25 \mathrm{~J}$ is shown in Fig.2(b). Remarkably, the anisotropy increases with doping in the underdoped regime, then decreases with doping and still have a ratio $\eta_{1}=1.2$ at the heavily overdoped regime $x=0.26$. This specific behavior is due to the variation of the topology of the Fermi surface with doping. Because this dependence is experimentally accessible, it will be interesting to compare the experimental result with our theoretical prediction, considering that the recent experiment was just carried out at the nearly optimal doped $\mathrm{YBa}_{2} \mathrm{Cu}_{3} \mathrm{O}_{6.85}$ 20].

Fig.3(a-c) show the intensity plot of the imaginary part of the spin susceptibility $\operatorname{Im} \chi^{s}$ as a function of the frequency and momentum. At the resonance frequency, the peak position is at $(\pi, \pi)$. Below and above the reso-
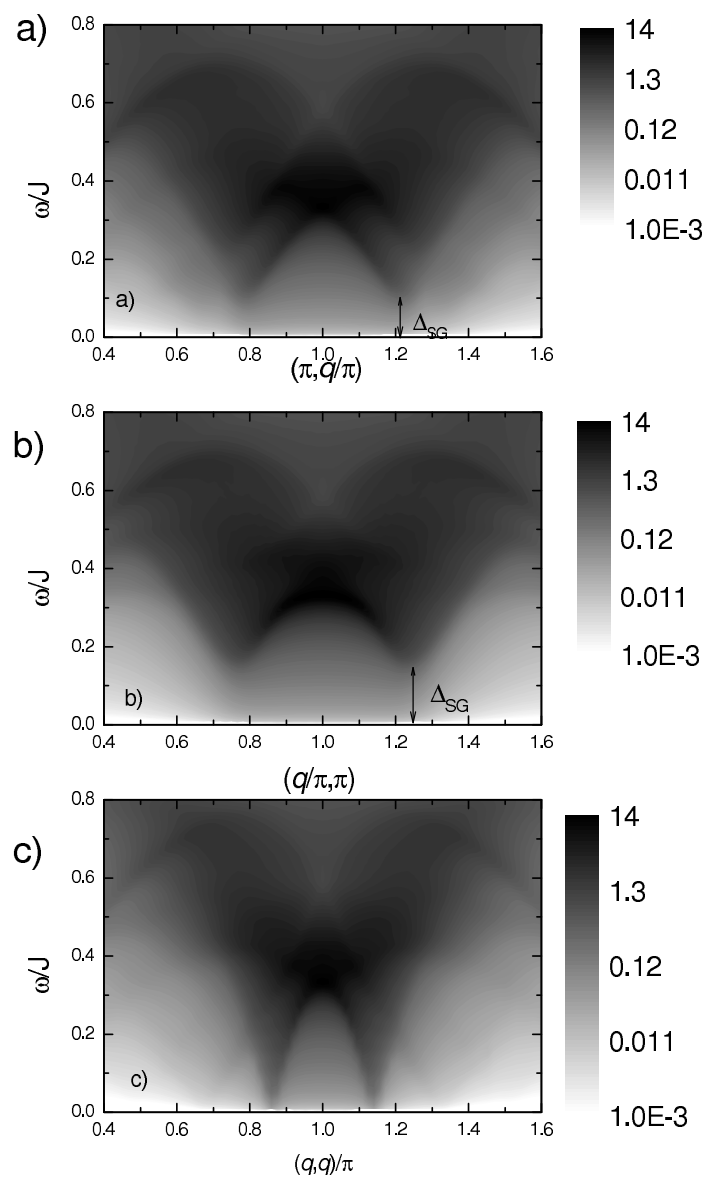

FIG. 3: Intensity plot of $\operatorname{Im} \chi^{s}$ as a function of frequency $\omega$ and wave vector $\mathbf{q}$. Panels a)-c) are along the $(\pi, q),(q, \pi)$ and $(q, q)$ direction, respectively.

nance frequency the IC peaks develop. The incommensurability is bigger as the frequency is gradually far away from the resonance frequency. Therefore, they exhibits a downward dispersion for frequency below the resonance frequency and an upward dispersion for frequency above the resonance frequency. This feature is consistent with the recent neutron scattering data 22, 23]. From Fig.3(a) and Fig.3(b), we can see an obvious spin gap at the $(q, \pi)$ and $(\pi, q)$ direction. Compared with that calculated for the system without the plane-chain coupling [15], a pronounced anisotropy in the spin gap is found, namely, the spin gap in the $(q, \pi)$ direction is bigger than that in the $(\pi, q)$ direction. Due to the existence of nodes, there is no spin gap along the diagonal direction as shown in Fig.3(c).

\section{B. dynamical charge susceptibility}

In Fig.4(a-c), we show the imaginary part of the charge susceptibility $\operatorname{Im} \chi^{c}$ as a function of the momentum with doping $x=0.14$ at frequencies $0.1 \mathrm{~J}, 0.3 \mathrm{~J}$, and $0.5 \mathrm{~J}$, respectively. An obvious feature of the charge susceptibil- 

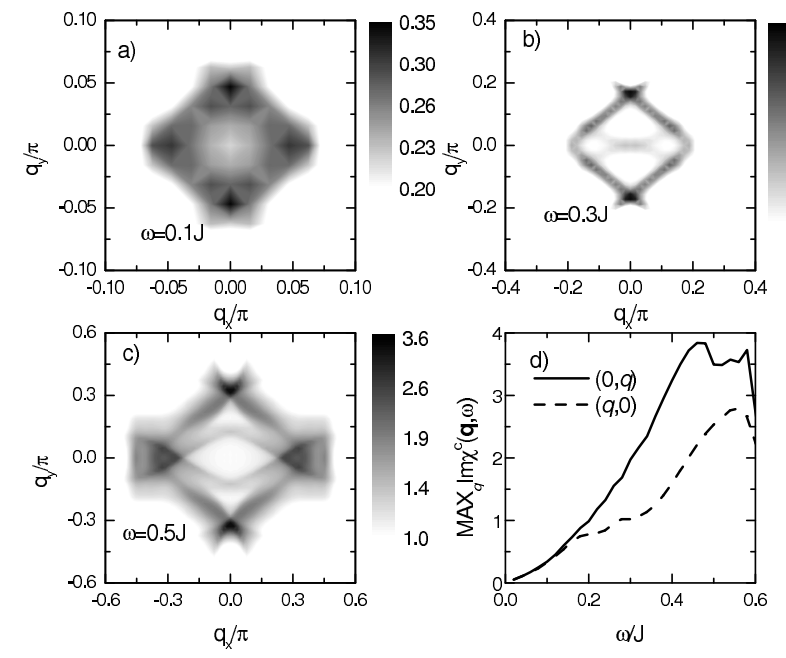

FIG. 4: Panels a)- c) are the intensity plot of the imaginary part of the charge susceptibility $\operatorname{Im} \chi^{c}$ as a function of wave vector with doping $x=0.14$ for $\omega=0.1 \mathrm{~J}, 0.3 \mathrm{~J}$ and $0.5 \mathrm{~J}$, respectively. Panel d) shows the frequency dependence of the maximal intensity of $\operatorname{Im} \chi^{c}$. The solid line denotes the result along the $(0, q)$ direction and the dashed line along the $(q, 0)$ direction.

ity is that its IC peak appears at small momenta around $(0,0)$, namely, at $(0, \delta)$ and $(\delta, 0)$. However, in sharp contrast to the anisotropy in the spin channel, the maximum IC peak in the charge channel is along the $k_{y}$ direction. Meanwhile, the IC peak is always along the $(0, \delta)$ and $(\delta, 0)$, while that in the spin channel will rotate to the diagonal direction at low and high frequencies. The frequency dependence of the IC peak intensity is shown in Fig.4(d). As shown, the anisotropy exists in a wide frequency range. It firstly appears at $\omega_{c}=0.1 \mathrm{~J}$ and becomes significant above $\omega=0.2 \mathrm{~J}$.

The intensity of the charge susceptibility as a function of the momentum and frequency is shown in Fig.5(a) and Fig.5(b). The charge susceptibility is incommensurate for all frequencies considered. The incommensurability will increase as the frequency increases and form a usual upward dispersion. This is quite different from the dispersion found in the spin channel, where the spin resonance at the commensurate $\mathrm{AF}$ wave vector $\mathbf{Q}=(\pi, \pi)$ disparts the dispersion into a downward form at low frequencies and an upward form at high frequencies. In the stripephase picture for the spin IC fluctuation [9], a change in the AF phase of the stripe at the charge-domain boundary occurs and it leads to a spin periodicity twice that of the charge periodicity. As a result, a two-to-one ratio of the incommensurability $\delta$ between the charge fluctuation and the spin fluctuation is expected. Here, though we can obtain the relation at a certain frequency $\omega=0.3 \mathrm{~J}$, generally there should be no such correspondence because of the different curvature of their dispersion. This feature comes from the topology of the Fermi surface and its origin will be discussed in the following section. It constitutes an additional difference between the Fermi-
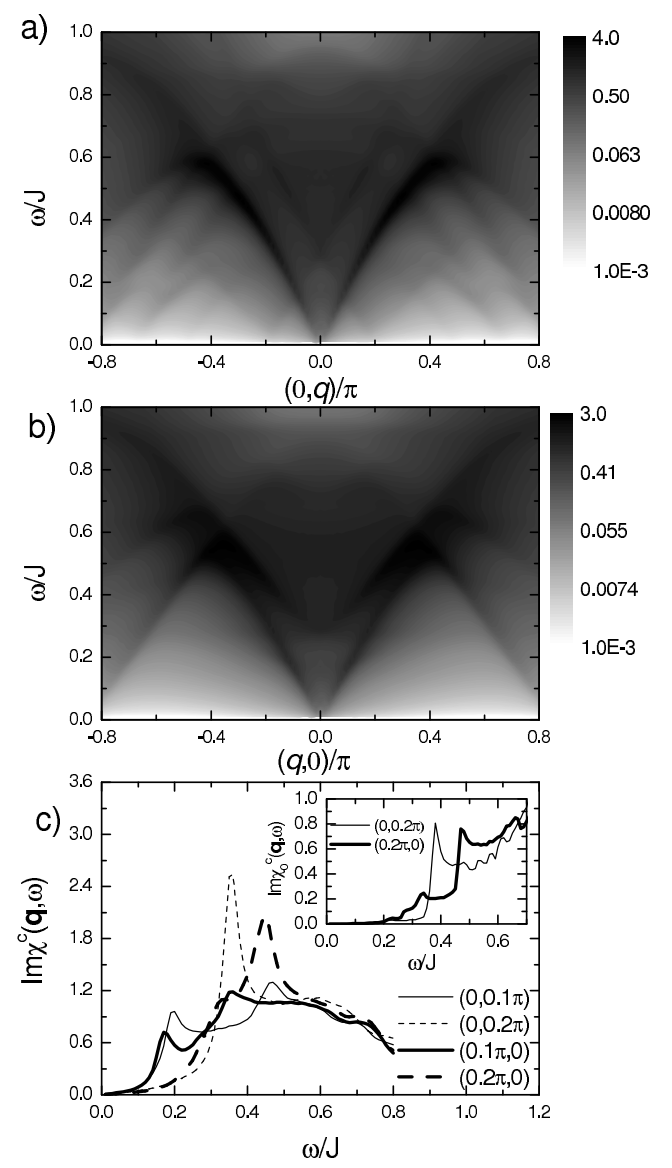

FIG. 5: Panels a) and b) are the intensity plot of $\operatorname{Im} \chi^{c}$ as a function of frequency $\omega$ and wave vector $\mathbf{q}$ along $(0, q)$ and $(q, 0)$ direction, respectively. Panel c) is the frequency dependence of $\operatorname{Im} \chi_{c}$ with different wave vectors. The inset of panel c) shows the frequency dependence of $\operatorname{Im} \chi_{0}^{c}$.

surface explanation and the stripe-phase explanation. In Fig.5(c), the imaginary part of the charge susceptibility as a function of the frequency is shown at small momentum transfers. For smaller momentum transfer $\mathbf{q}$, the charge excitation exhibits a broad spectrum. With the increase of $\mathbf{q}$, the lower energy spectrum is suppressed and a peak occurs at the edge of the gap. With the increase of $\mathbf{q}$, the peak intensity increases and reaches the maximum intensity around $|\mathbf{q}|=0.4 \pi$, as can be seen from Fig.5(a) and (b). From the inset of Fig.5(c) we can see that the imaginary part of the bare charge susceptibility also has a peak at the same frequency. Moreover, we have checked the pole equation $1-1 / 4 J_{\mathbf{q}} \chi_{0}^{c}(\mathbf{q}, \omega)=0$, and found that it does not satisfy at the peak energy. So, this peak is not the resonance peak as observed in the spin channel, rather it is right the IC charge peak. Thus, an anisotropy in the peak frequency and width exists in two different directions of the momentum transfer q, as shown in Fig.5(c). 


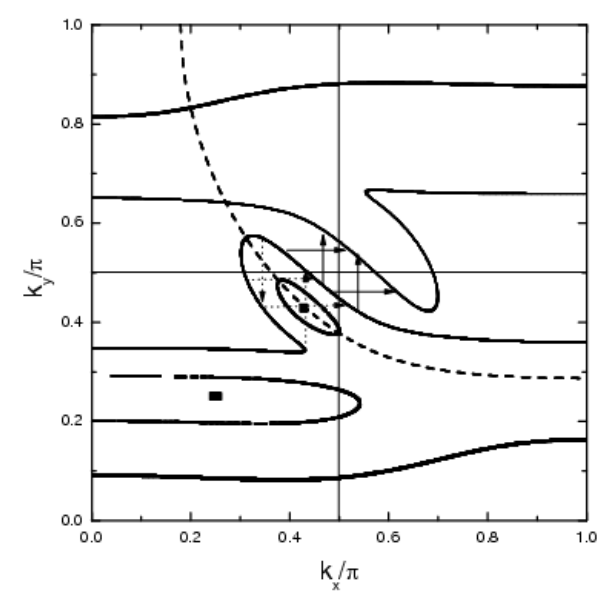

FIG. 6: Contour plot of the quasiparticle energy $E_{\mathbf{k}}=\omega / 2$ shown in the first quadrant of the Brillouin zone for $\omega=$ $0,0.12 J, 0.3 J$ and its by $(\pi, \pi)$ shifted image at $\omega=0.3 J$. The dashed line shows the normal state Fermi surface. The solid arrows denote the nesting vectors relative to $(\pi, \pi)$. The dotted arrows denote the nesting vectors within the energy contour.

\section{DISCUSSION}

Let us now explain the origin of the above features based on the topology of the Fermi surface. In the Fermi-liquid based theory, the spin and charge susceptibilities arise from the scattering of the SC quasiparticle across the SC gap. Their only difference is the sign difference in their coherence factors, namely, $c^{s(c)}=$ $1-\left(\varepsilon_{\mathbf{k}} \varepsilon_{\mathbf{k}+\mathbf{q}} \pm \Delta_{\mathbf{k}} \Delta_{\mathbf{k}+\mathbf{q}}\right) / E_{\mathbf{k}} E_{\mathbf{k}+\mathbf{q}}$, which is reflected by the plus/minus sign in front of $U_{1 i}$ in Eq.(5) and (7). Therefore, the coherence factor is appreciable when the gap $\Delta_{\mathbf{k}}$ and $\Delta_{\mathbf{k}+\mathbf{q}}$ have the opposite sign for the spin channel and the same sign for the charge channel. Thus, the peak of the imaginary part of the spin susceptibility will be near the $\mathrm{AF}$ wave vector $\mathbf{Q}=(\pi, \pi)$ because $\Delta_{\mathbf{k}}=-\Delta_{\mathbf{k}+\mathrm{Q}}$ and that of the charge susceptibility will be near the $(0,0)$ point. Then, the origin of the observed IC peaks can be illustrated in term of the initial and final scattering processes along energy contours, with different transfer wave vectors for the spin and the charge channel. Fig.6 shows the contour plot of the quasiparticle energy in the SC state $E_{\mathbf{k}}=\omega / 2$ at $\omega=0,0.12 J, 0.3 J$, and its by $(\pi, \pi)$ shifted image at $\omega=0.3 J$. In the spin channel, the transfer wave vector of the main scattering processes should be near $(\pi, \pi)$. Therefore, for an intermediate excitation energy such as $\omega=0.3 J$, the best nesting vector (solid arrows in Fig.6) will be a horizontal and vertical offset to $(\pi, \pi)$, which connects the flat part of the energy contour with its by $(\pi, \pi)$ shifted image. The scattering with this best nesting vector contributes directly to the IC peaks, therefore the IC peaks will appear in the parallel direction. In the case that there is no plane-chain coupling, the energy contour will be symmetric with re-
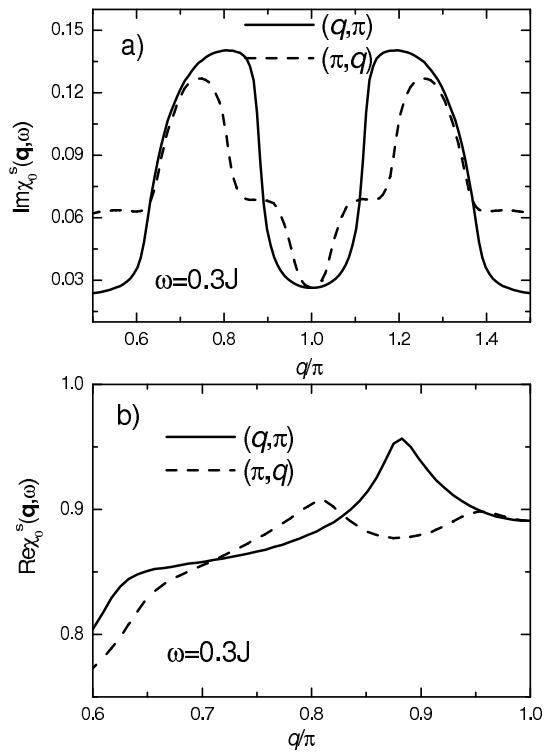

FIG. 7: Panel a) shows the imaginary part of the bare spin susceptibility as a function of wave vector with $\omega=0.3 \mathrm{~J}$. Panel b) is its real part.

spect to the diagonal direction. The coupling between the plane and chain will break this symmetry and the resulting nesting portion of the Fermi surface connected by the nesting vector along the $k_{x}$ direction is larger than that along the $k_{y}$ direction, as shown in Fig.6. So, both the imaginary and the real part of the bare spin susceptibility $\chi_{0}^{s}$ show an obvious anisotropy, i.e., the peak intensity at $\left(\pi \pm \delta_{x}, \pi\right)$ is stronger than that at $\left(\pi, \pi \pm \delta_{y}\right)$.

However, the anisotropy coming just from $\operatorname{Im} \chi_{0}^{s}$ is in fact small, as can be seen from a comparison between that in Fig.7(a) and Fig.1(d). The RPA correction factor $1+\alpha J_{\mathbf{q}} \operatorname{Re} \chi_{0}^{s}$ in the renormalized spin susceptibility $\operatorname{Im} \chi^{s}$ acts to enhance this anisotropy. The minimum of the RPA correction factor along the $(q, \pi)$ direction is less than that along the $(\pi, q)$ direction, because the maximum value of $\operatorname{Re} \chi_{0}^{s}$ along the former direction is larger than the latter direction, as shown in Fig7(b). As frequency approaches to the resonance frequency, $\operatorname{Re} \chi_{0}^{s}$ will tend to $-1 /\left(\alpha J_{\mathbf{q}}\right)$ and this makes the IC peak move close to $(\pi, \pi)$. Therefore, the anisotropy will become less and less and eventually vanish at the resonance energy. In addition, the RPA correction factor also affects strongly the incommensurability $\delta$ of the spin susceptibility. From Fig.7 (a), one can notice that the IC peak position in $\operatorname{Im} \chi_{0}^{s}$ along the $\left(\pi \pm \delta_{x_{0}}, \pi\right)$ direction is nearer to the $(\pi, \pi)$ point than that along the $\left(\pi, \pi \pm \delta_{y_{0}}\right)$ direction $\left(\delta_{x_{0}}<\delta_{y_{0}}\right)$. While in the renormalized spin susceptibility [shown in Fig.1(a)], this anisotropy of the incommensurability is reversed, i.e., $\delta_{x}>\delta_{y}$. In the experiment [20] one can also find that the peak position at $\left(\pi, \pi \pm \delta_{y}\right)$ is a little nearer to $(\pi, \pi)$ than that at $\left(\pi \pm \delta_{x}, \pi\right)$.

At low frequencies, the RPA correction factor plays minor role. The anisotropy of the spin gap along $k_{x}$ and $k_{y}$ direction is mainly determined by the anisotropy of 
the bare spin susceptibility. As frequency tends to zero, only node-to-node excitation becomes available, so there is no spin excitation along the $(q, \pi)$ and $(\pi, q)$ direction. As the frequency increases to $\omega_{y}=0.12 J$, the constant energy contour crosses the line $k_{x}=\pi$, as shown in Fig.6. So, the spin excitation along $(\pi, q)$ appears because now this constant energy contour and its by $(\pi, \pi)$ shift image [not shown in Fig.6] can be connected by the nesting wave vector along the $k_{y}$ direction. The critical frequency $\omega_{y}$ may be taken approximately as the spin gap along the $k_{y}$ direction. Because the constant energy contour does not cross the line $k_{y}=\pi$ at this frequency, there is still no spin excitation along the $k_{x}$ direction, i.e., the spin gap along the $k_{x}$ direction is bigger than that along the $k_{y}$ direction.

In the high energy region, the energy contour will evolve into two constant energy lines which are parallel approximately to the Fermi surface in the normal state, so the best nesting wave vector will rotate to the diagonal direction. In this case, the nesting wave vector along the $(\pi+\delta, \pi+\delta)$ and the $(\pi+\delta, \pi-\delta)$ direction is symmetric. Thus, the plane-chain coupling can not cause the anisotropy in the diagonal direction in the Fermi-liquid based theory.

In the charge channel, the transfer wave vector of the main scattering processes should be near $(0,0)$. Therefore, the best nesting vector (dotted arrows in Fig.6) is within the energy contour and contributes the IC peak at $(0, \delta)$ and $(\delta, 0)$. At low energies, the plane-chain coupling is less effective, so the constant energy contour is basically symmetric with respect to the diagonal direction and there is no anisotropy in the charge fluctuation. As the frequency increases, the plane-chain coupling destructs part of the nesting portion. It leads the nesting portion of the Fermi surface along the $k_{y}$ direction to be larger than the $k_{x}$ direction, so the IC peak along the $k_{y}$ direction is stronger than that along the $k_{x}$ direction.

\section{CONCLUSION}

In summary, we examine the frequency evolution of the spin and charge susceptibility based on the two di- mensional $t-t^{\prime}-J$ model considering the coupling between the plane and chain. In the spin channel, the IC peaks appear at $\left(\pi, \pi \pm \delta_{y}\right)$ and $\left(\pi \pm \delta_{x}, \pi\right)$ below the resonance frequency $(0.2 J<\omega<0.35 J)$. In this region, a pronounced anisotropy of the spin susceptibility is found, i.e., the peak intensity along the $k_{x}$ direction is stronger than that along the $k_{y}$ direction. Above the resonance frequency, the IC peaks reemerge and rotate to the diagonal direction, and there is no anisotropy in the IC peak intensity along the two diagonal directions. The dependence of the anisotropy on the antiferromagnetic correction factor $\alpha$ and the doping density $x$ is investigated, and it is found that the anisotropy is robust in the reasonable range. In addition, we also find an obvious anisotropy of the spin gap between the $(\pi, q)$ and the $(q, \pi)$ direction. The spin gap along the $k_{x}$ direction is bigger than that along the $k_{y}$ direction. In the charge channel, the susceptibility is also incommensurate for all energies we considered. The IC peaks appear at $(0, \pm \delta)$ and $( \pm \delta, 0)$. In sharp contrast to the spin channel, the IC peak intensity in the charge channel along the $k_{y}$ direction is stronger than that along the $k_{x}$ direction. Meanwhile, the IC peak exhibits an upward dispersion in the whole energy region, in contrast to the downward dispersion below the resonance frequency in the spin channel. In addition, no resonance mode is found in the charge channel. We explain all the results based on the scenario of the nesting Fermi surface and suggest that the coupling between the plane and chain is responsible for the observed anisotropy.

\section{Acknowledgments}

This work was supported by National Nature Science Foundation of China $(10474032,10021001)$ and by RFDP (20030284008).
[1] T. E. Mason, G. Aeppli, and H. A. Mook, Phys. Rev. Lett. 68, 1414 (1992).

[2] H. A. Mook, P. Dai, S. M. Hayden, G. Aeppli, T. G. Perring, and F. Doğan, Nature 395, 580 (1998).

[3] J. Rossat-Mignod, L. P. Regnault, C. Vettier, P. Bourges, P. Burlet, J. Bossy, J. Y. Henry, and G. Lapertot, Physica C 185-189, 86 (1991).

[4] J. M. Tranquada, P. M. Gehring, G. Shirane, S. Shamoto, and M. Sato, Phys. Rev. B 46, 5561 (1992).

[5] H. A. Mook, M. Yethiraj, G. Aeppli, T. E. Mason, and T. Armstrong, Phys. Rev. Lett 70, 3490 (1993).

[6] H. F. Fong, B. Keimer, P. W. Anderson, D. Reznik, F.
Doğan, and I. A. Aksay, Phys. Rev. Lett. 75, 316 (1995).

[7] H. A. Mook and F. Doğan, Nature (London) 401, 145 (1999).

[8] H. A. Mook, F. Doğan, and B. C. Chakoumakos, cond-mat/9811100 (1998) (unpublished).

[9] J. M. Tranquada, B.J. Sternlieb, J. D. Axe, Y. Nakamura, and S. Uchida, Nature 375, 561 (1995).

[10] V. J. Emery and S. A. Kivelson, Physica C 235-240, 189 (1994).

[11] J. Brinckmann and P. A. Lee, Phys. Rev. Lett. 82, 2915 (1999).

[12] D. K. Morr and D. Pines, Phys. Rev. B 61, 6483 (2000). 
[13] Y. J. Kao, Q. Si, and K. Levin, Phys. Rev. B 61, 11898 (2000).

[14] M. R. Norman, Phys. Rev. B 61, 14751 (2000).

[15] J. X. Li and C. D. Gong, Phys. Rev. B 66, 014506 (2002).

[16] J. X. Li, C. Y. Mou, and T. K. Lee, Phys. Rev. B 62, 640 (2000).

[17] J. X. Li, J. Zhang, and J. Luo, Phys. Rev. B 68, 224503 (2003).

[18] H. A. Mook, P. Dai, F. Doğan, and R. D. Hunt, Nature (London) 404, 729 (2000).

[19] T. Zhou and J. X. Li, Phys. Rev. B 69, 224514 (2004).

[20] V. Hinkov, S. Pailhes, P. Bourges, Y. Sisis, A. Ivanov, A. Kulakov, C. T. Lin, D. P. Chen, C. Bernhard, and B. Keimer, Nature 430, 650 (2004).

[21] We thank B. Keimer for pointing these points to us.

[22] S. M. Hayden, H. A. Mook, Pengcheng Dai, T. G. Per- ring, and F. Doğan, Nature 429, 531 (2004).

[23] J. M. Tranquada, H. Woo, T. G. Perring, H. Goka, G. D. Gu, G. Xu, M. Fujita, and K. Yamada, Nature 429, 534 (2004).

[24] I. Eremin and D. Manske, Phys. Rev. Lett. 94, 067006 (2005).

[25] Ying-Jer Kao and Hae-Young Kee, cond-mat/0412285 (2004) (unpublished).

[26] T. Xiang and J. M. Wheatley, Phys. Rev. Lett. 76, 134 (1996).

[27] W. A. Atkinson and J. P. Carbotte, Phys. Rev. B 52, 10601 (1995).

[28] H. Yamase, H. Kohno, H. Fukuyama, and M. Ogata, J. Phy. Soc. Jpn. 68, 1082 (1999). 